HEYCOCK AND NEVILLE : RÖNTGEN RAY

\title{
LXXVIII.-Röntgen Ray Photography Applied to Alloys.*
}

\section{By Charles Thomas Heycock and Francis Henry Neville.}

WHEN a salt solution is cooled until solid matter begins to form, the process of solidification may, if we exclude the contingency of the formation of definite crystalline hydrates, take place in one of three ways. Let us suppose that we are dealing with a solution of potassium permanganate, where the strong colouring matter of the salt makes the observation of the phenomena easy. Then, if the solidification follows a normal course, and for the sake of simplicity we shall suppose that it does so, we may observe the following cases.

a.-If a sufficiently dilute solution be slowly cooled, then at some temperature below zero colourless crystals of ice will begin to form, the whole of the permanganate remaining in solution in the mother liquor. As this residual liquid becomes more concentrated, the ice will form at a continually lower and lower temperature, but when, by the removal of the solvent as ice, a certain concentration of the permanganate has been reached, the permanganate, or conceivably a definite hydrate of it, will begin to solidify together with the water, and no further fall in temperature will be observed until everything has become solid. Thus the process of solidification consists of two stages.

b. - The second case occurs when a hot concentrated solution of permanganate is cooled. In this case, the first crystals that form will be pure permanganate, or conceivably a definite hydrate, and only after a considerable quantity of this has solidified at lower and lower temperatures, will ice begin to form, from this point onwards the solidification again taking place at a constant temperature until theiwhole is solid. The constant temperature during the second stage of $a$ is the same as that of the second stage of $b$, the concentration being also the same in both eases.

c.-From the above, it is obvious that a solution of permanganate can be prepared of such a strength that its solidification will take place from beginning to end at the constant temperature which marks the second stages of $a$ or $b$, the ice and the solid salt forming simultaneously. We may say that such a solution is saturated both with ice and with permanganate when at its freezing point, since, if we attempt to cool it further, both of these substances separate in the same proportion as that in which they are present in the liquid; solu-

* A brief preliminary notice of this paper was published in the Proceedings of the Chemical Society, 1897, p. 105. 
tions of the latter class were first investigated by Guthrie and termed by him "eutectic solutions." Guthrie at one time believed that such solutions and the solid matter formed from them were definite chemical compounds, but this view has since proved to be erroneous.

These three types of solidification can also be studied by the aid of a freezing point curve. When such a curve is traced by experiment for mixtures in every proportion of two substances, the freezing point, or halt in the cooling which marks the separation of solid matter, being taken as the ordinate, and the percentage composition being taken as the abscissa, we find the following to be the simplest case. A branch starts from the melting point of each of the pure substances, the two branches meeting in an angle at their lowest point; there is also a horizontal straight line passing through the intersection of the two branches. The points on one branch correspond to the first stage of $a$, those on the other to the first stage of $b$, whilst the angle corresponds to the case $c$, and the horizontal straight line through it corresponds to the second stages of $a$ and $b$.

Several observers have studied the solidification of alloys by plotting the freezing point curves, but, on account of the opacity of all alloys and of other difficulties, it has hitherto been impossible to follow and to study the formation of crystals as in the above-mentioned case of permanganate. The remarkable differences, however, in transparency to the Röntgen rays shown by different metals led us to hope that after slowly cooling a liquid alloy of two metals, one of high and the other of low atomic weight, we might be able to photograph through the solid alloy and examine the crystalline separations that had taken place. This method has proved so far of somewhat limited applicability, but in several cases we have been successful. The present paper contains results obtained with the following pairs of metals ; gold and sodium, gold and aluminium, copper and aluminium, silver and aluminium. The structure of solid alloys has usually been studied with the aid of the microscope on account of the minute character of the separations which occur, but as skiagraphs admit of but little subsequent magnification, it was indispensable for our purpose that the detail, that is to say the individual crystals in the alloy, should be large. We obtained this result by making the process of solidification a very gradual one ; an account of the cooling apparatus will be found at the end of the paper. The alloys were usually allowed to cool and solidify in cylindrical crucibles, the solid cylinders thus obtained being nearly an inch in diameter and from 2 to 4 inches long. Vertical and horizontal sections of these cylinders were cut, and when possible turned down to a thickness of less than a millimetre and polished. These sections were laid on an Ilford plate enclosed in a light-tight 
envelope, and exposed to the Röntgen rays for periods varying from five minutes to an hour.

The figures in the plate accompanying this paper (p. 722) correspond to positives, and therefore the lighter details indicate the most transparent metal. The figures are the natural size of the objects; they should be examined by means of a pocket lens.

\section{Gold-Sodium Alloys.}

In the Journal of the Chemical Society for 1889 (p. 668), we traced the changes in the freezing point of sodium caused by the addition of gold; we found that the eutectic alloy contained about 23 per cent. of gold and had a freezing point of $82^{\circ} \mathrm{C}$. Since these metals differ so much in their transparency to the Röntgen rays, it seemed well to commence with them.

To make the alloys, weighed quantities of sodium were melted and weighed quantities of gold added. The alloy was then heated, either under a mineral oil or in a sealed vacuous tube, with constant agitation, to a temperature sufficient to dissolve all the gold. Clean portions of the alloy were then sucked up with a pipette and transferred to a wide test-tube immersed vertically in a boiling brine bath, sufficient precautions being taken to avoid any danger of oxidation of the sodium, either by contact with the air or with moisture. The bath was kept constantly stirred by a current of air, and allowed to cool very slowly during a period of 24 hours or more. From the solid cylinder of alloy thus obtained, horizontal and vertical sections were cut, these sections being usually from 1 to 2 millimetres in thickness. Each section was immediately placed between sheets of very thin aluminium foil and the edges sealed by melted paraffin. Thus each section was in an air-tight cell and could usually be preserved for some weeks without serious oxidation.

All the sodium-gold alloys were photographed by means of a Crookes' tube excited by a Rhumkorff coil giving a 6-inch spark, the exposure varying from 5 to 30 minutes, according to the amount of gold present. The photographs 1 to 5 give the results of passing the Röntgen rays through sodium-gold.

Number 1 was a photograph from an alloy containing 3 per cent. of gold. It will be seen that the solid alloy is very far from being homogeneous, the bulk of the photograph consisting of light-coloured masses, strongly suggesting rectangular crystallisation, whilst the spaces between are filled with a much darker substance. Remembering that the photograph is a positive, we see that the larger and lighter part corresponds to a transparent substance which must therefore be mainly or entirely sodium, whilst the small quantity of dark interstitial matter 
must, from its opacity, contain all or nearly all the gold. As the photograph was produced by a shadow, the thickness of the plate of alloy appears to have somewhat blurred the outline of the crystalline forms.

We here appear to be dealing with a process of solidification very similar to that of case $a$ above referred to, a large amount of the solvent, in this case sodium, solidifying during the first stage of the cooling and leaving all the gold in a concentrated solution between the interstices of the crystals. During the second stage of the freezing, this eutectic alloy itself solidifies.

Figures 2 and 3 represent a similar phenomenon in the case of an alloy containing 10 per cent. of gold. The alloys were prepared in the same way but the sections cut were thinner, a fact which may account for the greater sharpness in the photographs. The opaque eutectic alloy necessarily occupies a larger space, but the contrast between the transparency of the crystals and the background is so strong that we are forced to the conclusion that the crystals of sodium contain but little, if any, gold. If we did not know from other grounds that the solvent sodium begins to solidify in these alloys before the gold, we could infer this from the straightness of the crystal lines, which in the photographs often exceed a centimetre in length. Both the transverse and longitudinal'sections of this alloy show a system of interlacing lines; we are disposed to think, however, that these indicate the existence of plates of sodium standing at right angles to the plane of the section, as this is the only structure that would account for so great a difference of transparency. If this view is correct, the alloy must possess a remarkable cellular structure somewhat similar to that which has been thought to exist in certain kinds of steel. We may mention that, when after some months we removed the sections from their aluminium cells, it was noticed that the slight oxidation of the surface had produced a pattern resembling that of the photographs.

Figure 4 is a photograph through an alloy containing more than 23 per cent. of gold. It was made by adding excess of gold to sodium at a temperature above $150^{\circ}$, and then slowly cooling as before. The photograph shows that the process of solidification resembles that of case $b$, for the large transparent crystals of sodium are absent, and we have instead a network of extremely opaque needles which consist of almost pure gold. These lie in a finely-grained matrix which must be the eutectic alloy containing nearly all the sodium and a portion of the gold.

If an alloy such as No. 4 is thrown into alcohol, the sodium is removed and the gold is left as a felted mass of crystalline needles. The needles, which are often 3 millimetres or more in length, appear to be square in section. They are so entirely different in character 
from the original gold used in making the alloy, that there can be no doubt of the gold having been completely in solution in the sodium. The gold used in making the alloy was prepared by precipitation with sulphur dioxide. Its crystals are very minute, but appear to be cubes or octahedra, needles being rarely or never present in it. In numerous analyses of the needles after removal of the excess of sodium by alcohol, we invariably found from 2 to 3 per cent. of sodium; to remove this sodium, it was necessary to ignite the crystals at a red heat and then extract for some time with water or alcohol. Thus it would seem that the substance of the needles contains a small amount of sodium.

Fig. 5 gives the result of photographing through the pure eutectic alloy. A transverse section was cut through the upper part of the same cylinder of alloy as that which furnished No. 4, the latter being cut from the middle of the cylinder. In 5 we see that the large black needles so conspicuous in 4 are almost absent, the reason being that as the gold began to crystallise from the fluid alloy it gradually sank towards the bottom, leaving a supernatant liquid of the composition of the eutectic alloy. In further confirmation fof this, we found that a section cut from near the base of the cylinder was much richer in the gold needles than even section 4 . In section 5, a few large crystals of sodium and of gold can still be seen, but the bulk of the alloy is much more uniform than in the other sections. Several attempts were made to prepare the eutectic alloy by weighing out gold and sodium in the exact eutectic proportion and heating the mixture in a sealed vacuous tube, but in most cases the photographs showed some large crystals of gold and sodium on a granular ground like that of No. 5. These photographs are of some interest, as the large crystals in what should be an eutectic alloy perhaps indicate supersaturation on the part of one or both metals, followed by rapid crystallisation. To save expense, however, No. 5 is the only case of an eutectic alloy reproduced in the paper.

The photographs show that the roughly uniform groundwork is, even in an eutectic alloy, not really homogeneous. Most probably, the gold and sodium separate side by side and simultaneously in very small crystals, although as the photographs admit of but little magnification, it is impossible to make out the separate crystals of the eutectic groundwork in such a section as No. 5. Osmond has, however, shown by a microscopic examination with a high power, that the eutectic alloy of silver and copper consists of white and red crystals; thus it is probable that eutectic alloys are as much conglomerates as the majority of other alloys, differing from them by the fineness of their grain and the greater uniformity of their composition. 
PHOTOGRAPHY APPLIED TO ALLOYS. View Article 719 ine

\section{Gold-Aluminium Alloys.}

Aluminium is nearly as transparent as sodium, and it is known that gold dissolves freely in aluminium; alloys of these two metals were therefore the next selected for examination. The discovery by Professor Roberts-Austen of the chemical compound $\mathrm{AuAl}_{2}$ formed by these two metals when melted together, and the great stability of this compound (Trans., 1894, 65, p. 75) give an especial interest to this group of alloys. The alloys were made in a small Fletcher's blast furnace and slowly cooled in the manner described in the introduction and the appendix.

Two alloys containing 5 and 20 per cent. of gold respectively were made and cooled simultaneously.

The 5 per cent. alloy of figure 6 shows the structure of an unsaturated solution of gold in aluminium, for the aluminium has crystallised first in transparent lines which are so straight that they must have been formed at an early stage of the freezing. The dark basis, as before, represents the finely grained eutectic alloy. From the method of making the alloy, we are convinced that it was quite homogeneous when fluid, but the photographs show that, as the result of slow cooling, there has been a distinct concentration of the gold towards the central portions of the ingot, which, of course, remained hot longest. The rectangular character of the aluminium crystallisation is even more marked than that of the sodium in the alloys containing that metal. Sections 6 and 7 are vertical sections through the upper and lower halves respectively of the cylinder of alloy, and section 8 is a horizontal one through the middle of the cylinder. Section 7 was inverted.

Fig. 9 is the photograph of a horizontal section near the bottom of an alloy containing 11 per cent. of gold. It will be noticed that whilst the bulk of the photograph is a mosaic of light and dark probably corresponding to the eutectic alloy, a few intensely opaque crystals are visible near the edge of the section. A careful examination of the polished surface of the alloy itself, with the aid of a lens, reveals these crystals as purple markings. They are, therefore, Roberts-Austen's alloy, $\mathrm{AuAl}_{2}$. From the small number of these crystals, we may conclude that the aluminium was not much more than saturated with this substance when the eutectic temperature was reached. Cryoscopic experiments have shown us that the eutectic alloy of gold and aluminium contains about $7 \cdot 1$ per cent. of gold, and an analysis of the upper middle part of the 20 per cent. gold alloy shows that this part contained $7 \cdot 4$ per cent. Au.

Although these photographs do not prove the point, we have reason 
to think that none of the gold is uncombined, and that the eutectic alloy when solid is a finely grained mixture of crystals of aluminium and of $\mathrm{AuAl}_{2}$. The outline of the opaque crystals strongly reminds one of those of potassium iodide. In Figs. 10 and 11 we have photographs of an alloy containing 20 per cent. of gold. Here, on account of the slowness of the cooling, the precipitated compound settles to the bottom as it forms, the upper part of the cylinder showing the coarsely mottled eutectic alloy, which is evidently far from being homogeneous, and the lower part affording an excellent picture of the crystals of the compound. In the negative of Fig. 10, some of the dark crystals appear to be octahedral, whilst in that of Fig. 11, which is a horizontal section near the bottom of the same cylinder, many appear to be cubical. A naked-eye examination of the surface of the section from which Fig. 10 is taken shows the brilliant purple of the compound.

We think that a careful examination of the upper part of Fig. 10, shows very well how completely heterogeneous an eutectic alloy may be, and that the fineness or coarseness of its grain is a mere function of the rate of cooling.

A striking contrast to these cases of slow cooling is presented by Fig. 12, which is the photograph of the same 20 per cent. gold alloy, but of a portion which has been quickly cooled by pouring on to a stone slab. It will be seen that the massive crystals are absent but that the whole alloy is full of minute black rods and rectangular crosses. These photographs illustrate very clearly the differences in structure that may exist between the same alloy when rapidly or when slowly cooled, and it is obvious that the mechanical properties would differ in a corresponding manner. Fig. $12 \mathrm{~A}$ is a section of the quickly cooled 5 per cent. gold alloy; it will be seen to have a very uniform grain.

\section{Copper-Aluminium Alloys.}

Figs. 13 to 16 are photographs of copper-aluminium alloys taken in the same manner as the preceding.

An alloy containing 5 per cent. of copper is shown in Fig. 13. Here the long straight lines of the aluminium crystals resemble those seen in the gold-aluminium alloy number 6 , and this might be expected, as they were formed under very similar conditions in the two cases. The darker eutectic alloy is, however, more uniformly distributed in the copper than in the gold alloy.

In Fig. 14, we have a horizontal section through the middle of a cylinder containing rather more than 14 per cent. of copper; several very perfect crosses of aluminium will be seen in this section, with a fern-like crystallisation starting from their arms. The dark eutectic alloy is here concentrated in one part of the section. 
Figs. 15 and 16 are from an alloy containing 25 per cent. of copper. The aluminium crystals are smaller and have a more fernlike appearance, and the mother substance, of course, occupies more space. In 16, which is a vertical section, we see, in addition, a few very opaque needles, which must be either copper or a compound of copper and aluminium.

The 50 per cent. copper alloy is so brittle that we have as yet failed in obtaining sections thin enough to be transparent.

\section{Silver-Aluminium Alloys.}

We have examined a number of slowly-cooled silver-aluminium alloys, but with the exception of the alloy $\mathrm{AgAl}_{4}$, of Figs. 17, 18, and 19 , we have not obtained as yet details in the X-ray photographs distinct enough for reproduction. Fig. 17 is a horizontal section, and 18 and 19 are vertical sections.

Slowly-cooled silver-zinc alloys, although the polished surfaces show abundant detail when examined by the naked eye or with a lens, show no detail in the photographs. The same is true of zinc-gold alloys.

We have obtained very fine photographs from alloys of aluminium with either antimony, nickel, or platinum. We hope to discuss and reproduce these in a continuation of this paper.

\section{Method of Cooling.}

In order to produce the very slow cooling required to make the alloys give the maximum separation, it was necessary to devise a special arrangement for slowly and continuously cutting off the gas from the furnace. The apparatus for this purpose is represented in the accompanying sketch, p. 722 (not drawn to scale); it is seen to consist essentially of four parts.

The constant level water dropper, $A$, worked very steadily, and obviated the difficulty caused by small gas bubbles collecting in the tap, and so stopping the flow of water. By measuring the water which drops into a graduated cylinder for one minute, and knowing the capacity of the cylinder $B$, we can ascertain the rate at which the gas will be cut off. The zinc cylinder $B$ is about 4 feet high and 10 inches in diameter, and holds some 55 litres; it is provided with a water gauge and emptying tube as shown in the diagram; it has also an overflow at the top. This zinc cylinder is connected by a rubber tube with the narrow necked bottle $C$, of about 250 c.c. capacity. This bottle, which should be rather wide in proportion to its height, has a hole drilled in its bottom, into which a glass tube is cemented. The $\operatorname{tap} C^{\prime}$ is for the purpose of getting rid of air bubbles which are liable 
to collect in $C$. Joined to $C$ by a thick-walled rubber tube is the gas cutoff $D$; the tube $D$ is $15 \mathrm{~cm}$. long and $2.5 \mathrm{~cm}$. wide, and it contains an inner tube made of fifteen layers of fine silk wound round the outside of a cylinder of coarse iron wire gauze in order to give it rigidity. The silk tube is open at its lower end, and is fastened at its upper end to the glass tube $D^{\prime \prime}$, by the aid of cement. With a gas pressure of about 1 inch of water, the silk tube, even with 15 layers, allows more gas to pass than

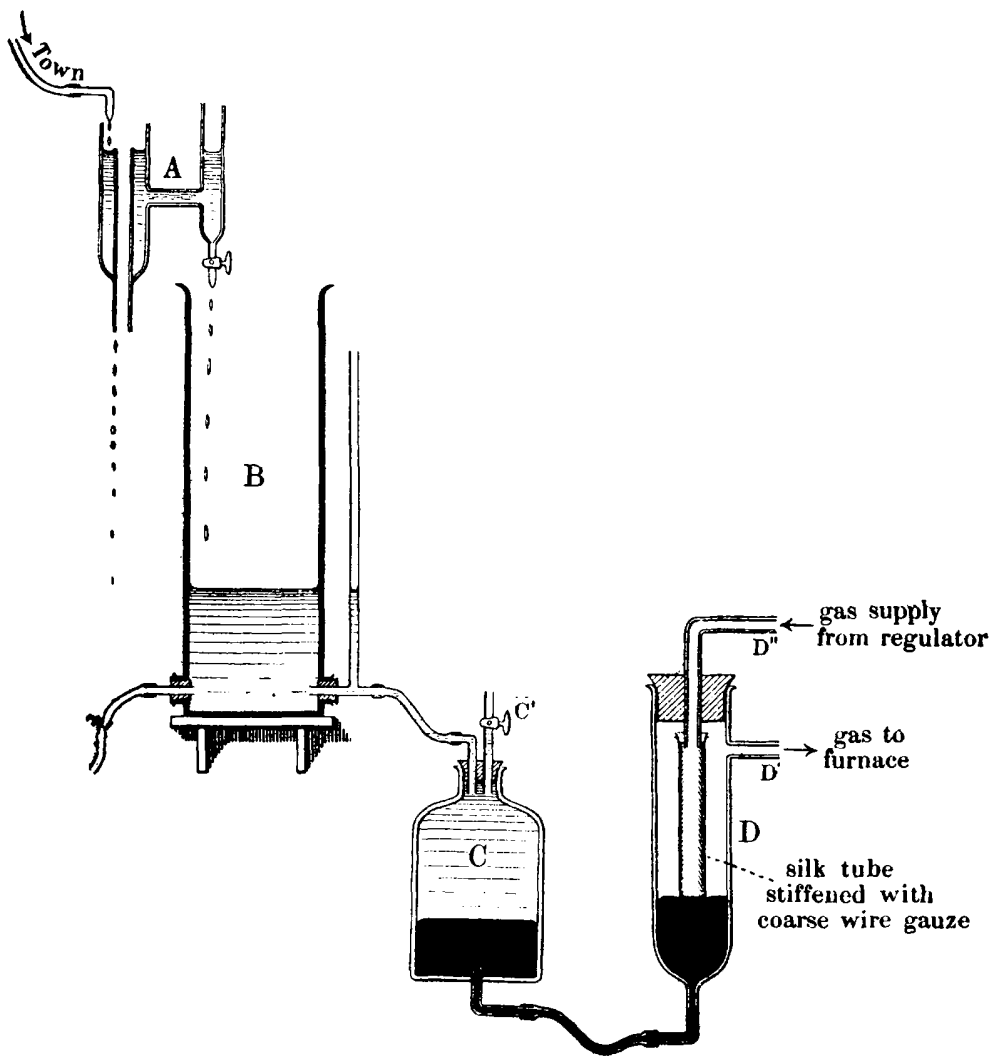

is required to keep a Fletcher's draught furnace (No. 363) up to a temperature of the melting point of silver. This difficulty was got over by entirely coating the silk tube with a very viscous shellac, except for a line about $2 \mathrm{~mm}$. wide running from top to bottom of the silk. It will be seen by the aid of the diagram that, as the water drips into $B$, the pressure on the surface of the mercury in $C$ slowly and steadily increases, thus causing the mercury to flood the tube $D$ 


\section{Plate I.}
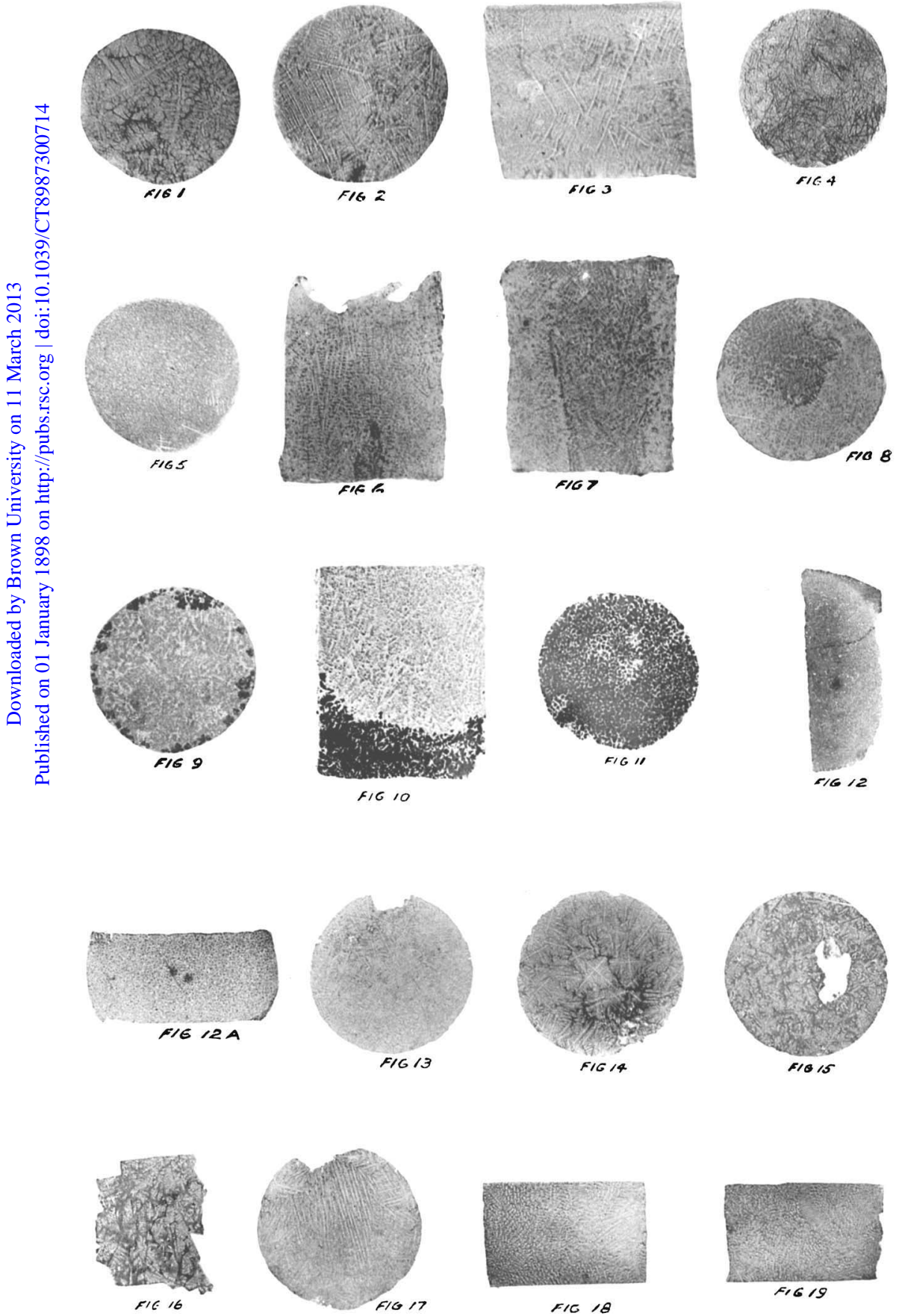
both inside and out, and hence to gradually cut off the escape of gas through the unvarnished portion.

Such a cut-off is useless unless the gas pressure is kept constant. The pressure regulator we have used was designed by Mr. Horace Darwin, and supplied by the Cambridge Scientific Instrument Company. Careful measurements have shown that this regulator controls the very great variations of gas pressure we have in Cambridge, passing, as they do, from a minimum of about $1 \frac{3}{4}$ inches to a maximum of about 3 inches; it also overcomes the changes of pressure produced by conducting other furnace operations simultaneously in the laboratory. The regulator has the additional advantage that it can be loaded with weights so as to deliver gas to the burner at any desired pressure up to the minimum of the town supply. In order to obtain the greatest possible steadiness during the cooling, we place a large clay crucible in the furnace, and set within it two or three small salamander cylinders of about 22 c.c. capacity, the interstices between the cylinders being filled in with sand. The furnace is heated to the highest temperature required, and the water dropper and cut-off set working. The alloys are then melted and thoroughly stirred in a blast furnace, poured into the salamander cylinders, and allowed to cool down; this process of cooling usually occupies from 40 to 50 hours. When dealing with alloys which melt at a high temperature we can, by aid of a bye-pass, allow sufficient gas to pass direct to the furnace to keep it at the lowest temperature required, the cut-off thus becoming more efficient.

Sinnky Colifege,

Cambitinge. 